\title{
Comments on fixed point results in classes of function with values in a $b$-metric space
}

\author{
Janusz Brzdęk ${ }^{1}$ \\ Received: 14 July 2020 / Accepted: 9 October 2021 / Published online: 2 November 2021 \\ (c) The Author(s) 2021
}

\begin{abstract}
We prove and discuss several fixed point results for nonlinear operators, acting on some classes of functions with values in a b-metric space. Thus we generalize and extend a recent theorem of Dung and Hang (J Math Anal Appl 462:131-147, 2018), motivated by several outcomes in Ulam type stability. As a simple consequence we obtain, in particular, that approximate (in some sense) eigenvalues of some linear operators, acting in some function spaces, must be eigenvalues while approximate eigenvectors are close to eigenvectors with the same eigenvalue. Our results also provide some natural generalizations and extensions of the classical Banach Contraction Principle.
\end{abstract}

Keywords b-metric · Fixed point · Function space · Ulam stability · Approximate eigenvalue

Mathematics Subject Classification 39B82 - 47A63 - 47H10 - 47J99 · 54H25

\section{Introduction}

The notion of Ulam stability (see $[8,16,22]$ for details) has motivated several generalizations of Banach Contraction Principle for various function spaces (see, e.g., [3,4,6,7,9]). Let us recall for instance the main result in [9] (see also [4, Theorem 2]). To formulate it we need the following hypothesis for operators $\Lambda: \mathbb{R}_{+}{ }^{E} \rightarrow \mathbb{R}_{+}{ }^{E}$ ( $\mathbb{R}_{+}$stands for the set of nonnegative reals, $E$ is a nonempty set and $A^{B}$ denotes the family of all functions mapping a nonempty set $B$ into a nonempty set $A$ ):

$\left(\mathcal{C}_{0}\right)$ If $\left(\delta_{n}\right)_{n \in \mathbb{N}}$ is a sequence in $\mathbb{R}_{+}{ }^{E}$ with $\lim _{n \rightarrow \infty} \delta_{n}(t)=0$ for $t \in E$, then

$$
\lim _{n \rightarrow \infty}\left(\Lambda \delta_{n}\right)(t)=0, \quad t \in E .
$$

Janusz Brzdęk

brzdek@agh.edu.pl

1 Faculty of Applied Mathematics, AGH University of Science and Technology, Mickiewicza 30, 30-059 Kraków, Poland 
Let us yet remind that, given a metric space $(Y, \rho), \emptyset \neq \mathcal{C} \subset Y^{E}$ and an operator $\Lambda: \mathbb{R}_{+}{ }^{E} \rightarrow \mathbb{R}_{+}{ }^{E}$, we say that $\mathcal{T}: \mathcal{C} \rightarrow Y^{E}$ is $\Lambda$-contractive (cf., e.g., [9]) if

$$
d((\mathcal{T} v)(t),(\mathcal{T} \mu)(t)) \leq(\Lambda \delta)(t), \quad t \in E,
$$

for any $\nu, \mu \in \mathcal{C}$ and $\delta \in \mathbb{R}_{+}{ }^{E}$ such that

$$
d(v(t), \mu(t)) \leq \delta(t), \quad t \in E .
$$

Next, if $A$ is a nonempty set and $F \in A^{A}$, then $F^{0}$ is the identity function on $A$ (i.e., $F^{0}(x)=x$ for $\left.x \in A\right)$ and

$$
F^{n+1}=F \circ F^{n}, \quad n \in \mathbb{N}_{0}:=\mathbb{N} \cup\{0\},
$$

where $\mathbb{N}$ stands for the set of positive integers.

The main result in [9] can be formulated as follows (see [7] for an abstract generalization of it).

Theorem 1 [9, Theorem 2.2] Let $(Y, \rho)$ be a complete metric space, $E$ be a nonempty set, operator $\Lambda: \mathbb{R}_{+}{ }^{E} \rightarrow \mathbb{R}_{+}{ }^{E}$ fulfil $\left(\mathcal{C}_{0}\right), \mathcal{T}: Y^{E} \rightarrow Y^{E}$ be $\Lambda$-contractive, and $\varepsilon: E \rightarrow \mathbb{R}_{+}$ and $\varphi: E \rightarrow Y$ be such that

$$
\rho((\mathcal{T} \varphi)(t), \varphi(t)) \leq \varepsilon(t), \quad \sigma(t):=\sum_{n \in \mathbb{N}_{0}}\left(\Lambda^{n} \varepsilon\right)(t)<\infty, \quad t \in E .
$$

Then the limit

$$
\psi(t):=\lim _{n \rightarrow \infty}\left(\mathcal{T}^{n} \varphi\right)(t)
$$

exists for each $t \in E$ and the function $\psi \in Y^{E}$, defined in this way, is a unique fixed point of $\mathcal{T}$ with

$$
\rho\left(\left(\mathcal{T}^{n} \varphi\right)(t), \psi(t)\right) \leq \sum_{i=n}^{\infty}\left(\Lambda^{i} \varepsilon\right)(t), \quad t \in E, n \in \mathbb{N}_{0} .
$$

Moreover, if $\lim _{n \rightarrow \infty}\left(\Lambda^{n} \sigma\right)(t)=0$ for $t \in E$, then $\psi$ is the unique fixed point of $\mathcal{T}$ with

$$
\rho(\varphi(t), \psi(t)) \leq \sigma(t), \quad t \in E .
$$

A very interesting extension of the theorem to the b-metric spaces, but only for $\Lambda$ of a particular form, has been presented by Dung and Hang [13, Theorem 2.1]. Let us recall that a b-metric, in a nonempty set $Y$, is a function $d: Y \times Y \rightarrow \mathbb{R}_{+}$satisfying the following three conditions:

(A) $d(x, y)=0$ if and only if $x=y$;

(B) $d(x, y)=d(y, x)$;

(C) $d(x, y) \leq \eta(d(x, z)+d(z, y))$

for all $x, y, z \in Y$ and some real constant $\eta \geq 1$. If conditions (A)-(C) are valid, then we say that $(Y, d, \eta)$ is a b-metric space. Let us yet mention that the $\mathrm{b}$-metric spaces also have been named quasi-metric spaces by some authors (see, e.g., [15]), which is better corresponding to the notion of quasi-norms (see Remark 6). However, the term $b$-metric seems to be more convenient, as the name quasi-metric has also other meanings (see, e.g, [12] and [27]); for instance in [12] it means a function $d$ satisfying only (A) and (C) with $\eta=1$ (without (B)). 
The term $b-$ metric has been used for the first time in [10] with $\eta=2$ and next in [11] for any $\eta \geq 1$.

Let us yet remind that, in the b-metric spaces, the notions of convergence, Cauchy sequence and a limit of a sequence are defined analogously as in the classical metric spaces. Namely, if $(Y, d, \eta)$ is a b-metric space, then:

- a sequence $\left(x_{n}\right)_{n \in \mathbb{N}} \in Y^{\mathbb{N}}$ is convergent to $x \in Y$ if $\lim _{n \rightarrow \infty} d\left(x, x_{n}\right)=0$ and then we say that $x$ is a limit (which must be unique) of the sequence and write $x=\lim _{n \rightarrow \infty} x_{n}$;

- a sequence $\left(x_{n}\right)_{n \in \mathbb{N}} \in Y^{\mathbb{N}}$ is Cauchy if $\lim _{n, m \rightarrow \infty} d\left(x_{m}, x_{n}\right)=0$;

- $(Y, d)$ is complete if every Cauchy sequence in $Y$ is convergent to some point $x \in Y$.

The result in [13] can be formulated in the following way.

Theorem 2 [13, Theorem 2.1] Let $U$ be a nonempty set, $(Y, d, \eta)$ be a complete b-metric space, and $\mathcal{T}: Y^{U} \rightarrow Y^{U}$. Assume that there exist $\varepsilon: U \rightarrow \mathbb{R}_{+}, \varphi: U \rightarrow Y, f_{1}, \ldots, f_{k}:$ $U \rightarrow U$ and $L_{1}, \ldots, L_{k}: U \rightarrow \mathbb{R}_{+}$such that

$$
\begin{aligned}
& d((\mathcal{T} v)(t),(\mathcal{T} \mu)(t)) \leq \sum_{i=1}^{k} L_{i}(t) d\left(v\left(f_{i}(t)\right), \mu\left(f_{i}(t)\right)\right), \quad v, \mu \in Y^{U}, t \in U, \\
& d((\mathcal{T} \varphi)(t), \varphi(t)) \leq \varepsilon(t), \quad \varepsilon^{*}(t)=\sum_{n=0}^{\infty}\left(\left(\Lambda^{n} \varepsilon\right)(t)\right)^{\theta}<\infty, \quad t \in U,
\end{aligned}
$$

where $\theta=\log _{2 \eta} 2$ and $\Lambda: \mathbb{R}_{+}{ }^{E} \rightarrow \mathbb{R}_{+}{ }^{E}$ is given by

$$
(\Lambda \delta)(t):=\sum_{i=1}^{k} L_{i}(t) \delta\left(f_{i}(t)\right), \quad \delta \in \mathbb{R}_{+}^{U}, t \in U .
$$

Then limit (1) exists for every $t \in U$ and the function $\psi: U \rightarrow Y$ so defined is a fixed point of $\mathcal{T}$ with

$$
d(\varphi(t), \psi(t))^{\theta} \leq 4 \varepsilon^{*}(t), \quad t \in U
$$

Moreover, if

$$
\varepsilon^{*}(t) \leq\left(M \sum_{n=1}^{\infty}\left(\Lambda^{n} \varepsilon\right)(t)\right)^{\theta}<\infty, \quad t \in U,
$$

with some real number $M>0$, then $\psi$ is the unique fixed point of $\mathcal{T}$ satisfying (4).

Remark 3 Note that every operator of form (3) fulfils hypothesis $\left(\mathcal{C}_{0}\right)$.

Remark 4 Let $r \in(1, \infty)$ and $(Z, d)$ be a metric space. Then $\left(Z, d_{r}, 2^{r-1}\right)$ is a b-metric space, where $d_{r}(x, y):=d(x, y)^{r}$ for $x, y \in Z$. In fact, for every $x, y, z \in Z$,

$$
\begin{aligned}
d_{r}(x, y) & \leq(d(x, z)+d(z, y))^{r} \\
& \leq 2^{r-1}\left(d(x, z)^{r}+d(z, y)^{r}\right)=2^{r-1}\left(d_{r}(x, z)+d_{r}(z, y)\right),
\end{aligned}
$$

because $(a+b)^{r} \leq 2^{r-1}\left(a^{r}+b^{r}\right)$ for $a, b \in \mathbb{R}_{+}$.

The reasoning presented in Remark 4 also leads to the following two examples (cf. [2, Examples 1.1 and 1.2]). 
Example 1 Let $X$ be a Banach space, $p \in(0,1)$,

$$
\ell_{p}(X):=\left\{\left(x_{n}\right)_{n \in \mathbb{N}} \in X^{\mathbb{N}}: \sum_{n=1}^{\infty}\left\|x_{n}\right\|^{p}<\infty\right\}
$$

and $\rho: \ell_{p}(X)^{2} \rightarrow \mathbb{R}_{+}$be defined by

$$
\rho(x, y):=\left(\sum_{n=1}^{\infty}\left\|x_{n}-y_{n}\right\|^{p}\right)^{1 / p}, \quad x=\left(x_{n}\right)_{n \in \mathbb{N}}, y=\left(y_{n}\right)_{n \in \mathbb{N}} \in \ell_{p}(X) .
$$

Then $\left(\ell_{p}(X), \rho, 2^{(1-p) / p}\right)$ is a $b$-metric space.

Example 2 If $p \in(0,1)$,

$$
L_{p}[0,1]:=\left\{x:[0,1] \rightarrow \mathbb{R}: \int_{0}^{1}|x(t)|^{p} d t<1\right\},
$$

and $\rho: L_{p}[0,1] \times L_{p}[0,1] \rightarrow \mathbb{R}_{+}$is defined by

$$
\rho(x, y):=\left(\int_{0}^{1}|x(t)-y(t)|^{p} d t\right)^{1 / p}, \quad x, y \in L_{p}[0,1]
$$

then $\left(L_{p}[0,1], \rho, 2^{(1-p) / p}\right)$ is a $b$-metric space.

Moreover, note that if $n \in \mathbb{N}, a_{1}, \ldots, a_{n} \in(0, \infty)$ and $d_{1}, \ldots, d_{n}$ are b-metrics in a nonempty set $X$, then $d$ and $d_{0}$ also are b-metrics in $X$, where

$$
d(x, y)=\sum_{i=1}^{n} a_{i} d_{i}(x, y), \quad d_{0}(x, y)=\max _{i=1, \ldots, n} a_{i} d_{i}(x, y), \quad x, y \in X .
$$

Let us yet recall the following very useful result.

Theorem 5 [24, Proposition, p. 4308] Let $(Y, d, \eta)$ be a $b$-metric space and

$$
D_{d}(x, y)=\inf \left\{\sum_{i=1}^{n} d^{\xi}\left(x_{i}, x_{i+1}\right): x_{2}, \ldots, x_{n} \in Y, n \in \mathbb{N}, x_{1}=x, x_{n+1}=y\right\}
$$

for all $x, y \in Y$, where $\xi:=\log _{2 \eta} 2$ and $d^{\xi}(x, y)=(d(x, y))^{\xi}$ for $x, y \in Y$. Then $D_{d}$ is a metric in $Y$ with

$$
\frac{1}{4} d^{\xi}(x, y) \leq D_{d}(x, y) \leq d^{\xi}(x, y), \quad x, y \in Y .
$$

In particular, if $d$ is a metric, then $D_{d}=d$.

Remark 6 Assume that $(X,\|\cdot\|, \eta)$ is a quasi-normed space, i.e., $\eta \geq 1$ is a fixed real number, $X$ is a real or complex linear space and $\|\cdot\|: X \rightarrow \mathbb{R}_{+}$satisfies the following three conditions:

(a) $\|x\|=0$ if and only if $x=0$;

(b) $\|\alpha x\|=|\alpha|\|x\|$ for $x \in X$ and all scalars $\alpha$;

(c) $\|x+y\| \leq \eta(\|x\|+\|y\|)$ for $x, y \in X$.

Let $Y \subset X$ be nonempty and $d: Y^{2} \rightarrow \mathbb{R}_{+}$be given by: $d(x, y):=\|x-y\|$ for $x, y \in Y$. Then it is easily seen that $(Y, d, \eta)$ is a b-metric space. 
In view of Aoki-Rolewicz Theorem (see, e.g., [20, Theorem 1]), each quasi-norm is equivalent to some $p$-norm, but there exist $p$-Banach spaces which are not normable (see, e.g., [20, Examples 1 and 2]). Let us yet recall that, if $X$ is a a real (complex, respectively) linear space and $p>0$, then a mapping $\|\cdot\|: X \rightarrow \mathbb{R}_{+}$is a $p$-norm provided conditions (a) and (b) hold and

(c1) $\|x+y\|^{p} \leq\|x\|^{p}+\|y\|^{p}$ for $x, y \in X$.

In the next section we provide some generalizations of Theorem 2 by showing that similar fixed point results hold also for operators $\Lambda$ of more general forms. In this way we extend Theorem 1 and several similar outcomes in $[4-6,9]$ to the case of $b-$ metric spaces.

Finally, let us mention that in $[14,19,23,25,28,29]$ and references contained therein, the interested readers can find various further recent metric-type fixed point results. Maybe some of them could be extended and applied in a similar way as in this paper. It seems that the concept of $v$-generalized metric is very promising in this respect (see, e.g., [28]).

\section{The main result}

In what follows, we always assume that $(Y, d, \eta)$ is a complete b-metric space. Moreover, $E$ denotes a nonempty set and, given $v, \mu \in Y^{E}$, we define a mapping $d(v, \mu): E \rightarrow \mathbb{R}_{+}$by the formula:

$$
d(\nu, \mu)(t):=d(v(t), \mu(t)), \quad v, \mu \in Y^{E}, t \in E .
$$

Similarly, as in the usual metric spaces, if $\left(\chi_{n}\right)_{n \in \mathbb{N}}$ is a sequence in $Y^{E}$, then we say that $\chi \in Y^{E}$ is a pointwise limit of $\left(\chi_{n}\right)_{n \in \mathbb{N}}$ when

$$
\lim _{n \rightarrow \infty} d\left(\chi, \chi_{n}\right)(t)=0, \quad t \in E
$$

$\chi \in Y^{E}$ is a uniform limit of $\left(\chi_{n}\right)_{n \in \mathbb{N}}$ if

$$
\lim _{n \rightarrow \infty} \sup _{t \in E} d\left(\chi, \chi_{n}\right)(t)=0 .
$$

We say that a subset $\mathcal{F} \neq \emptyset$ of $Y^{E}$ is p-closed (u-closed, respectively) if each $\chi \in Y^{E}$ that is a pointwise (uniform, resp.) limit of a sequence of elements of $\mathcal{F}$, is an element of $\mathcal{F}$.

Further, if $f, g \in \mathbb{R}^{E}$, then we write $f \leq g$ provided $f(t) \leq g(t)$ for $t \in E$.

First note that from Theorem 1 we can easily deduce the following partial generalization of Theorem 2 (i.e., of [13, Theorem 2.1]), in which we do not need to presume any particular form of $\Lambda$, but has to replace (4) with a somewhat different estimate (see the definition of $\varepsilon^{*}$ in (2)) and formulate the statement on uniqueness similarly as in Theorem 1.

Theorem 7 Let $\Lambda: \mathbb{R}_{+}{ }^{E} \rightarrow \mathbb{R}_{+}{ }^{E}$ fulfil $\left(\mathcal{C}_{0}\right), \mathcal{T}: Y^{E} \rightarrow Y^{E}$ be $\Lambda$-contractive, and $\varepsilon: E \rightarrow$ $\mathbb{R}_{+}$and $\varphi: E \rightarrow Y$ be such that

$$
d((\mathcal{T} \varphi)(t), \varphi(t)) \leq \varepsilon(t), \quad \sum_{k \in \mathbb{N}_{0}}\left(\Lambda_{\xi}^{k} \varepsilon^{\xi}\right)(t)<\infty, \quad t \in E,
$$

where $\xi:=\log _{2 \eta} 2$ and $\varepsilon^{\xi}: E \rightarrow \mathbb{R}_{+}, \Lambda_{\xi}: \mathbb{R}_{+}{ }^{E} \rightarrow \mathbb{R}_{+}{ }^{E}$ are given by

$$
\varepsilon^{\xi}(t):=(\varepsilon(t))^{\xi}, \quad\left(\Lambda_{\xi} \delta\right)(t)=((\Lambda \delta)(t))^{\xi}, \quad \delta \in \mathbb{R}_{+}^{E}, t \in E .
$$


Then the limit

$$
\psi(t):=\lim _{n \rightarrow \infty}\left(\mathcal{T}^{n} \varphi\right)(t)
$$

exists for each $t \in E$ and the function $\psi \in Y^{E}$, defined in this way, is a unique fixed point of $\mathcal{T}$ with

$$
d\left(\mathcal{T}^{n} \varphi(t), \psi(t)\right)^{\xi} \leq 4 \sum_{k=n}^{\infty}\left(\left(\Lambda_{\xi}^{k} \varepsilon^{\xi}\right)(t)\right)^{\xi}, \quad t \in E, n \in \mathbb{N}_{0}
$$

Proof According to Theorem 5, (7) and the $\Lambda$-contractivity of $\mathcal{T}$,

$$
\begin{aligned}
D_{d}((\mathcal{T} \varphi)(t), \varphi(t)) & \leq d((\mathcal{T} \varphi)(t), \varphi(t))^{\xi} \leq \varepsilon^{\xi}(t) \\
D_{d}((\mathcal{T} v)(t),(\mathcal{T} \mu)(t)) & \leq d((\mathcal{T} v)(t),(\mathcal{T} \mu)(t))^{\xi} \\
& \leq((\Lambda d(v, \mu))(t))^{\xi}=\left(\Lambda_{\xi} d(\nu, \mu)\right)(t)
\end{aligned}
$$

for every $v, \mu \in Y^{E}$ and $t \in E$. Moreover, note that the completeness of $(Y, d, \eta)$ implies the completeness of the metric space $\left(Y, D_{d}\right)$ (see (6)). So, by Theorem 5 and Theorem 1 (with $\rho=D_{d}$ and $\Lambda$ and $\varepsilon$ replaced by $\Lambda_{\xi}$ and $\varepsilon^{\xi}$ ), we obtain the statement.

The next fixed point theorem provides a further, a bit different, generalization of Theorem 2 ; it is the main result of this paper. To simplify some formulas, we denote by $\Lambda_{0}$ the identity operator on $\mathbb{R}_{+}{ }^{E}$, i.e., $\Lambda_{0} \delta=\delta$ for all $\delta \in \mathbb{R}_{+}{ }^{E}$.

Theorem 8 Let $\mathcal{C} \subset Y^{E}$ be nonempty, $\mathcal{T}: \mathcal{C} \rightarrow \mathcal{C}, \Lambda_{n}: \mathbb{R}_{+}{ }^{E} \rightarrow \mathbb{R}_{+}{ }^{E}$ for $n \in \mathbb{N}$, and $\mathcal{T}^{n}$ be $\Lambda_{n}$-contractive for $n \in \mathbb{N}$. Suppose there are $\varepsilon \in \mathbb{R}_{+}{ }^{E}$ and $\varphi \in \mathcal{C}$ with

$$
d(\mathcal{T} \varphi, \varphi) \leq \varepsilon, \quad \varepsilon^{*}(t):=\sum_{i=0}^{\infty}\left(\left(\Lambda_{i} \varepsilon\right)(t)\right)^{\xi}<\infty, \quad t \in E,
$$

and one of the following two hypotheses is valid.

(i) $\mathcal{C}$ is p-closed.

(ii) $\mathcal{C}$ is $u$-closed and the sequence $\left(\sum_{i=0}^{n}\left(\Lambda_{i} \varepsilon\right)^{\xi}\right)_{n \in \mathbb{N}}$ tends uniformly to $\varepsilon^{*}$.

Then, for each $t \in E$, the limit

$$
\psi(t):=\lim _{n \rightarrow \infty}\left(\mathcal{T}^{n} \varphi\right)(t)
$$

exists and the function $\psi \in Y^{E}$, so defined, belongs to $\mathcal{C}$ and fulfils the inequalities

$$
d\left(\mathcal{T}^{n} \varphi, \psi\right) \leq\left(4 \sum_{i=n}^{\infty}\left(\Lambda_{i} \varepsilon\right)^{\xi}\right)^{1 / \xi}=: \varepsilon_{n}^{*}, \quad n \in \mathbb{N}_{0} .
$$

Moreover, the following three statements are valid.

(a) If $l \in \mathbb{N}$ and

$$
\liminf _{n \rightarrow \infty}\left(\Lambda_{l} \varepsilon_{n}^{*}\right)(t)=0, \quad t \in E,
$$

then $\psi$ is a fixed point of operator $\mathcal{T}^{l}$. 
(b) If $l \in \mathbb{N}, t \in E$ and $\gamma \in \mathbb{R}_{+}{ }^{E}$ are such that

$$
\liminf _{n \rightarrow \infty}\left(\Lambda_{\ln } \gamma\right)(t)=0
$$

then $\psi(t)=\omega(t)$ for every fixed point $\omega \in \mathcal{C}$ of $\mathcal{T}^{l}$, satisfying the inequality

$$
d(\varphi, \omega) \leq \gamma
$$

(c) If $t \in E$ and $\varepsilon_{n}^{*}(t) \neq 0$ for $n \in \mathbb{N}$, then $\tau(t)=\psi(t)$ for every $\tau \in \mathcal{C}$ with

$$
\liminf _{n \rightarrow \infty} \frac{d\left(\left(\mathcal{T}^{n} \varphi\right)(t), \tau(t)\right)}{\varepsilon_{n}^{*}(t)}<\infty .
$$

Proof Let $D_{d}$ be the metric defined as in Theorem 5. Since $\mathcal{T}^{n}$ is $\Lambda_{n}$-contractive, (8) implies that

$$
d\left(\mathcal{T}^{n+1} \varphi, \mathcal{T}^{n} \varphi\right) \leq \Lambda_{n} \varepsilon, \quad n \in \mathbb{N}
$$

whence

$$
\begin{aligned}
D_{d}\left(\mathcal{T}^{n+k} \varphi, \mathcal{T}^{n} \varphi\right) & \leq \sum_{i=0}^{k-1} D_{d}\left(\mathcal{T}^{n+i+1} \varphi, \mathcal{T}^{n+i} \varphi\right) \\
& \leq \sum_{i=0}^{k-1} d\left(\mathcal{T}^{n+i+1} \varphi, \mathcal{T}^{n+i} \varphi\right)^{\xi} \\
& \leq \sum_{i=n}^{n+k-1}\left(\Lambda_{i} \varepsilon\right)^{\xi} \leq \sum_{i=n}^{\infty}\left(\Lambda_{i} \varepsilon\right)^{\xi}
\end{aligned}
$$

and consequently

$$
\begin{aligned}
d\left(\mathcal{T}^{n+k} \varphi, \mathcal{T}^{n+r} \varphi\right)^{\xi} & \leq 4 D_{d}\left(\mathcal{T}^{n+k} \varphi, \mathcal{T}^{n+r} \varphi\right) \\
& \leq 4 D_{d}\left(\mathcal{T}^{n+k} \varphi, \mathcal{T}^{n} \varphi\right)+4 D_{d}\left(\mathcal{T}^{n} \varphi, \mathcal{T}^{n+r} \varphi\right) \\
& \leq 4 \sum_{i=n}^{\infty}\left(\Lambda_{i} \varepsilon\right)^{\xi}+4 \sum_{i=n}^{\infty}\left(\Lambda_{i} \varepsilon\right)^{\xi}
\end{aligned}
$$

for any $k, r \in \mathbb{N}$ and $n \in \mathbb{N}_{0}$, where $\left(\Lambda_{i} \varepsilon\right)^{\xi}(t):=\left(\left(\Lambda_{i} \varepsilon\right)(t)\right)^{\xi}$ for $t \in E$. So, by (8), $\left(\mathcal{T}^{n} \varphi(t)\right)_{n \in \mathbb{N}}$ is a Cauchy sequence in $(Y, d, \eta)$ for each $t \in E$, which means that it is convergent. Consequently, (9) defines a function $\psi \in Y^{E}$. Clearly, if (i) holds, then $\psi \in \mathcal{C}$.

Note that (9) holds also in the metric space $\left(Y, D_{d}\right)$, in view of (6). So, letting $k \rightarrow \infty$ in (15), for each $t \in E$ we get

$$
\begin{aligned}
d\left(\psi(t),\left(\mathcal{T}^{n} \varphi\right)(t)\right)^{\xi} & \leq 4 D_{d}\left(\psi(t),\left(\mathcal{T}^{n} \varphi\right)(t)\right) \\
& \leq 4 \sum_{i=n}^{\infty}\left(\left(\Lambda_{i} \varepsilon\right)(t)\right)^{\xi}, \quad n \in \mathbb{N}_{0},
\end{aligned}
$$

which is (10). Since, in the case of (ii), we have

$$
\lim _{n \rightarrow \infty} \sup _{t \in E}\left(\sum_{i=n}^{\infty}\left(\left(\Lambda_{i} \varepsilon\right)(t)\right)^{\xi}\right)=0,
$$


it is easily seen that (10) implies that $\psi \in \mathcal{C}$ also in this case.

Next, observe that (16) implies

$$
d\left(\mathcal{T}^{n+l} \varphi, \mathcal{T}^{l} \psi\right) \leq \Lambda_{l}\left(\left(4 \sum_{i=n}^{\infty}\left(\Lambda_{i} \varepsilon\right)^{\xi}\right)^{1 / \xi}\right)=\Lambda_{l} \varepsilon_{n}^{*}
$$

for $l, n \in \mathbb{N}_{0}$. So, for each $l, n \in \mathbb{N}_{0}$,

$$
\begin{aligned}
d\left(\psi, \mathcal{T}^{l} \psi\right) & \leq \eta d\left(\psi, \mathcal{T}^{n+l} \varphi\right)+\eta d\left(\mathcal{T}^{n+l} \varphi, \mathcal{T}^{l} \psi\right) \\
& \leq \eta d\left(\psi, \mathcal{T}^{n+l} \varphi\right)+\eta \Lambda_{l} \varepsilon_{n}^{*} .
\end{aligned}
$$

Hence, if (11) is valid for a fixed $l \in \mathbb{N}$, then with $n \rightarrow \infty$ we get

$$
d\left(\psi(t),\left(\mathcal{T}^{l} \psi\right)(t)\right)=0, \quad t \in E,
$$

and consequently $\mathcal{T}^{l} \psi=\psi$.

Now, let $l \in \mathbb{N}, \gamma: E \rightarrow \mathbb{R}_{+}$and $\omega \in \mathcal{C}$ be a fixed point of $\mathcal{T}^{l}$ satisfying (13). Then, for each $n \in \mathbb{N}$, we get

$$
\begin{aligned}
d(\psi, \omega) & =d\left(\psi, \mathcal{T}^{l n} \omega\right) \\
& \leq \eta d\left(\psi, \mathcal{T}^{l n} \varphi\right)+\eta d\left(\mathcal{T}^{l n} \varphi, \mathcal{T}^{l n} \omega\right)
\end{aligned}
$$

whence

$$
d(\psi(t), \omega(t)) \leq \eta d\left(\psi(t),\left(\mathcal{T}^{\ln } \varphi\right)(t)\right)+\eta\left(\Lambda_{\ln } \gamma\right)(t), \quad t \in E .
$$

Consequently, letting $n \rightarrow \infty$, by (9), we can easily see that $\omega(t)=\psi(t)$ for each $t \in E$ such that (12) holds.

Finally, let $t \in E$ and $\tau \in \mathcal{C}$ be such that $\varepsilon_{n}^{*}(t) \neq 0$ for $n \in \mathbb{N}$ and (14) is valid. Write

$$
M_{t}:=1+\liminf _{n \rightarrow \infty} \frac{d\left(\left(\mathcal{T}^{n} \varphi\right)(t), \tau(t)\right)}{\varepsilon_{n}^{*}(t)} .
$$

Then there is a strictly increasing sequence $\left(t_{n}\right)_{n \in \mathbb{N}}$ in $\mathbb{N}$ such that

$$
d\left(\left(\mathcal{T}^{t_{n}} \varphi\right)(t), \tau(t)\right)^{\xi}<\left(M_{t} \varepsilon_{t_{n}}^{*}(t)\right)^{\xi}=M_{t}^{\xi} \sum_{i=t_{n}}^{\infty}\left(\left(\Lambda_{i} \varepsilon\right)(t)\right)^{\xi}, \quad n \in \mathbb{N}
$$

and consequently, by (16),

$$
\begin{aligned}
d(\tau(t), \psi(t))^{\xi} & \leq 4 D_{d}\left(\tau(t),\left(\mathcal{T}^{t_{n}} \varphi\right)(t)\right)+4 D_{d}\left(\left(\mathcal{T}^{t_{n}} \varphi\right)(t), \psi(t)\right) \\
& \leq 4 d\left(\tau(t),\left(\mathcal{T}^{t_{n}} \varphi\right)(t)\right)^{\xi}+4 d\left(\left(\mathcal{T}^{t_{n}} \varphi\right)(t), \psi(t)\right)^{\xi} \\
& <4\left(M_{t}^{\xi}+1\right) \sum_{i=t_{n}}^{\infty}\left(\left(\Lambda_{i} \varepsilon\right)(t)\right)^{\xi}, \quad n \in \mathbb{N},
\end{aligned}
$$

whence we get $\tau(t)=\psi(t)$ with $n \rightarrow \infty$ (in view of (8)).

Remark 9 From statement (b) of Theorem 8 we can deduce, in particular, the following: If $\psi$ is not a fixed point of $\mathcal{T}^{l}$ for some $l \in \mathbb{N}$, then the same is true for every function $\omega \in \mathcal{C}$, which satisfies (13) with some $\gamma \in \Gamma_{l}$, where $\Gamma_{l}$ denotes the family of all functions $\gamma: E \rightarrow \mathbb{R}_{+}$ such that (12) holds for each $t \in E$. 
But, if $\psi$ is a fixed point of $\mathcal{T}^{l}$ for some $l \in \mathbb{N}$, then

$$
\psi(t)=\lim _{n \rightarrow \infty}\left(\mathcal{T}^{l k_{n}} \chi\right)(t)
$$

for every $\chi \in \mathcal{C}, t \in E$, and every sequence $\left(k_{n}\right)_{n \in \mathbb{N}}$ in $\mathbb{N}$ such that there is $\gamma \in \mathbb{R}_{+}{ }^{E}$ with $d(\chi, \psi) \leq \gamma$ and

$$
\lim _{n \rightarrow \infty}\left(\Lambda_{l k_{n}} \gamma\right)(t)=0 .
$$

In fact, if $\gamma: E \rightarrow \mathbb{R}_{+}$and $\chi \in \mathcal{C}$ satisfies the inequality $d(\chi, \psi) \leq \gamma$, then for each $t \in E$ and each sequence $\left(k_{n}\right)_{n \in \mathbb{N}}$ in $\mathbb{N}$ such that (17) holds, we have

$$
\begin{aligned}
d\left(\left(\mathcal{T}^{l k_{n}} \chi\right)(t), \psi(t)\right) & =d\left(\left(\mathcal{T}^{l k_{n}} \chi\right)(t),\left(\mathcal{T}^{l k_{n}} \psi\right)(t)\right) \\
& \leq\left(\Lambda_{l k_{n}} \gamma\right)(t), \quad n \in \mathbb{N},
\end{aligned}
$$

which implies that

$$
\psi(t)=\lim _{n \rightarrow \infty}\left(\mathcal{T}^{l k_{n}} \chi\right)(t)
$$

To avoid any ambiguity in the sequel, we need one more definition.

Definition 10 Let $(D, \rho, \eta)$ be a b-metric space, $T$ be a nonempty subset of $D, S: T \rightarrow D$ and $\lambda: \mathbb{R}_{+} \rightarrow \mathbb{R}_{+}$. We say that $S$ is a $\lambda$-contraction provided

$$
\rho(S(t), S(s)) \leq \lambda(\delta)
$$

for every $t, s \in T$ and every $\delta \in \mathbb{R}_{+}$such that $\rho(t, s) \leq \delta$.

It is clear that, for nondecreasing $\lambda, S$ is a $\lambda$-contraction if and only if

$$
\rho(S(t), S(s)) \leq \lambda(\rho(t, s)), \quad t, s \in T .
$$

Note yet that, if $E$ has only one element, i.e., $E=\left\{t_{0}\right\}$, then $Y^{E}$ can be identified with $Y$. Therefore, Theorem 8 yields at once the following generalization of the Banach Contraction Principle.

Corollary 11 Let $T: Y \rightarrow Y$ and $\lambda_{n}: \mathbb{R}_{+} \rightarrow \mathbb{R}_{+}$for $n \in \mathbb{N}$. Suppose that $T^{n}$ is a $\lambda_{n^{-}}$ contraction for $n \in \mathbb{N}$ and there exist $e \in \mathbb{R}_{+}$and $\phi \in Y$ such that

$$
\sum_{i=1}^{\infty} \lambda_{i}(e)^{\xi}<\infty, \quad d(T(\phi), \phi) \leq e .
$$

Then there exists the limit

$$
\psi_{0}:=\lim _{n \rightarrow \infty} T^{n}(\phi)
$$

and

$$
d\left(T^{n}(\phi), \psi_{0}\right) \leq\left(4 \sum_{i=n}^{\infty} \lambda_{i}(e)^{\xi}\right)^{1 / \xi}=: e_{n}^{*}, \quad n \in \mathbb{N}_{0},
$$

where $\lambda_{0}(d)=d$ for $d \in \mathbb{R}_{+}$. Moreover, the following statements are valid.

( $\alpha$ ) If $l \in \mathbb{N}$ and

$$
\liminf _{n \rightarrow \infty} \lambda_{l}\left(e_{n}^{*}\right)=0,
$$

then $\psi_{0}$ is a fixed point of $T^{l}$. 
( $\beta$ ) If $e_{0} \in \mathbb{R}_{+}, l \in \mathbb{N}$ and

$$
\liminf _{n \rightarrow \infty} \lambda_{\ln }\left(e_{0}\right)=0,
$$

then every fixed point $\phi_{0} \in Y$ of $T^{l}$, satisfying the inequality

$$
d\left(\phi, \phi_{0}\right) \leq e_{0},
$$

is equal to $\psi_{0}$.

( $\gamma$ ) If $e_{n}^{*} \neq 0$ for $n \in \mathbb{N}$, then $\psi_{0}$ is the unique element of the set

$$
\left\{z \in Y: \liminf _{n \rightarrow \infty} \frac{d\left(\left(T^{n}(\phi), z\right)\right.}{e_{n}^{*}}<\infty\right\} .
$$

Remark 12 Analogously as in Remark 9, from statement $(\beta)$ of Corollary 11 we can deduce, in particular, the following: If $\psi_{0}$ is not a fixed point of $T^{l}$ for some $l \in \mathbb{N}$, then the same is true for each $z \in E$ with $d\left(\psi_{0}, z\right)<\sup D$, where $D$ is the set of all $c \in \mathbb{R}_{+}$such that there is a sequence $\left(k_{n}\right)_{n \in \mathbb{N}}$ in $\mathbb{N}$ with

$$
\lim _{n \rightarrow \infty} k_{n}=\infty, \quad \liminf _{n \rightarrow \infty} \lambda_{l k_{n}}(c)=0 .
$$

However, if $\psi_{0}$ is a fixed point of $T^{l}$ for some $l \in \mathbb{N}$, then (again analogously as in Remark 9) it is easy to show that

$$
\psi_{0}=\lim _{n \rightarrow \infty} T^{l k_{n}}(v)
$$

for every $v \in E$ and every sequence $\left(k_{n}\right)_{n \in \mathbb{N}}$ in $\mathbb{N}$ such that there is $d \in \mathbb{R}_{+}{ }^{E}$ with $d\left(v, \psi_{0}\right) \leq$ $d$ and

$$
\lim _{n \rightarrow \infty} \lambda_{l k_{n}}(d)=0
$$

Theorem 8 also yields the subsequent.

Corollary 13 Let $\mathcal{C} \subset Y^{E}$ be nonempty, $\Lambda: \mathbb{R}_{+}{ }^{E} \rightarrow \mathbb{R}_{+}{ }^{E}, \mathcal{T}: \mathcal{C} \rightarrow \mathcal{C}$ be $\Lambda$-contractive, and functions $\varepsilon \in \mathbb{R}_{+}{ }^{E}$ and $\varphi \in \mathcal{C}$ be such that

$$
d(\mathcal{T} \varphi, \varphi) \leq \varepsilon, \quad \varepsilon^{\star}(t):=\sum_{i=0}^{\infty}\left(\left(\Lambda^{i} \varepsilon\right)(t)\right)^{\xi}<\infty, \quad t \in E .
$$

Next, assume one of the following two hypotheses.

(i) $\mathcal{C}$ is p-closed.

(ii) $\mathcal{C}$ is $u$-closed and the sequence $\left(\sum_{i=0}^{n}\left(\Lambda^{i} \varepsilon\right)^{\xi}\right)_{n \in \mathbb{N}}$ tends uniformly to $\varepsilon^{\star}$.

Then, for each $t \in E$, there exists the limit

$$
\psi(t):=\lim _{n \rightarrow \infty}\left(\mathcal{T}^{n} \varphi\right)(t)
$$

and the function $\psi \in Y^{E}$, so defined, belongs to $\mathcal{C}$ and fulfils the inequalities

$$
d\left(\mathcal{T}^{n} \varphi, \psi\right) \leq\left(4 \sum_{i=n}^{\infty}\left(\Lambda^{i} \varepsilon\right)^{\xi}\right)^{1 / \xi}=: \varepsilon_{n}^{\star}, \quad n \in \mathbb{N}_{0} .
$$

Moreover, the following statements are valid. 
(A) If

$$
\liminf _{n \rightarrow \infty}\left(\Lambda \varepsilon_{n}^{\star}\right)(t)=0, \quad t \in E,
$$

then $\psi$ is a fixed point of $\mathcal{T}$.

(B) If $t \in E$ and $\gamma \in \mathbb{R}_{+}{ }^{E}$ are such that

$$
\liminf _{n \rightarrow \infty}\left(\Lambda^{n} \gamma\right)(t)=0
$$

then $\psi(t)=\omega(t)$ for every fixed point $\omega \in \mathcal{C}$ of $\mathcal{T}$, satisfying the inequality $d(\varphi, \omega) \leq \gamma$.

(C) If $t \in E$ and $\varepsilon_{n}^{\star}(t) \neq 0$ for $n \in \mathbb{N}$, then $\tau(t)=\psi(t)$ for every $\tau \in \mathcal{C}$ with

$$
\liminf _{n \rightarrow \infty} \frac{d\left(\left(\mathcal{T}^{n} \varphi\right)(t), \tau(t)\right)}{\varepsilon_{n}^{\star}(t)}<\infty .
$$

Proof It is easy to see that $\mathcal{T}^{n}$ is $\Lambda^{n}$-contractive for each $n \in \mathbb{N}$. So, it is enough to use Theorem 8 with $l=1$ and $\Lambda_{n}:=\Lambda^{n}$ for $n \in \mathbb{N}$.

Remark 14 Fix $j \in \mathbb{N}$. Let $f_{i}: E \rightarrow E$ and $L_{i}: E \rightarrow \mathbb{R}_{+}$for $i=1, \ldots, j$. Define $\Lambda: \mathbb{R}_{+}{ }^{E} \rightarrow \mathbb{R}_{+}{ }^{E}$ by the formula

$$
\Lambda \delta(t):=\sum_{i=1}^{j} L_{i}(t) \delta\left(f_{i}(t)\right), \quad \delta \in \mathbb{R}_{+}{ }^{E}, t \in E .
$$

Then $\left(\mathcal{C}_{0}\right)$ holds for such $\Lambda$ (see Remark 3 ). This means that Theorem 1 is a simple consequence of Corollary 13. We show that this is also the case for Theorem 2.

First note that $\left(\mathcal{C}_{0}\right)$ and (18) imply (21). Therefore the statement of Theorem 2 that $\psi$ is a fixed point of $\mathcal{T}$ can be deduced from (A). We are yet to show that the statement on uniqueness of $\psi$ can be derived from (B).

To this end fix $\gamma \in \mathbb{R}_{+}{ }^{E}$ and $M>0$ such that

$$
\begin{gathered}
\gamma_{0}^{*}(t):=\left(\sum_{k=0}^{\infty}\left(\left(\Lambda^{k} \gamma\right)(t)\right)^{\xi}\right)^{1 / \xi}<\infty, \quad t \in E, \\
\gamma_{0}^{*}(t) \leq M \sum_{i=0}^{\infty}\left(\Lambda^{i} \gamma\right)(t), \quad t \in E .
\end{gathered}
$$

We prove that, for each $n \in \mathbb{N}_{0}$,

$$
\left(\Lambda^{n} \gamma_{0}^{*}\right)(t) \leq M \sum_{i=n}^{\infty}\left(\Lambda^{i} \gamma\right)(t), \quad t \in E .
$$

Clearly, the case $n=0$ is trivial in view of (23). Next, fix $n \in \mathbb{N}_{0}$ and suppose that (24) is valid. Then

$$
\begin{aligned}
\left(\Lambda^{n+1} \gamma_{0}^{*}\right)(t) & =\sum_{i=1}^{j} L_{i}(t)\left(\Lambda^{n} \gamma_{0}^{*}\right)\left(f_{i}(t)\right) \leq \sum_{i=1}^{j} L_{i}(t) M \sum_{k=n}^{\infty}\left(\Lambda^{k} \gamma\right)\left(f_{i}(t)\right) \\
& \leq M \sum_{k=n}^{\infty} \sum_{i=1}^{j} L_{i}(t)\left(\Lambda^{k} \gamma\right)\left(f_{i}(t)\right) \\
& =M \sum_{k=n}^{\infty}\left(\Lambda^{k+1} \gamma\right)(t)=M \sum_{k=n+1}^{\infty}\left(\Lambda^{k} \gamma\right)(t), \quad t \in E
\end{aligned}
$$


Thus, by induction, we have proved (24). Therefore, in this case, (24) yields (22). Consequently, (22) results also from the condition

$$
\gamma_{0}^{*}(t) \leq M \sum_{k=1}^{\infty}\left(\Lambda^{k} \gamma\right)(t), \quad t \in E .
$$

This means that (5) (cf. [13, (2.6)]) implies that

$$
\liminf _{n \rightarrow \infty}\left(\Lambda^{n} \varepsilon_{0}^{*}\right)(t)=0, \quad t \in E,
$$

and therefore from (B) we can infer that $\psi$ is the unique fixed point of $\mathcal{T}$, satisfying the inequality $d(\varphi, \psi) \leq \varepsilon_{0}^{*}$.

Thus we have shown that Theorem 2 (i.e., [13, Theorem 2.1]) is a simple consequence of Corollary 13.

The next example shows that, in some natural situations, assumption (8) can be significantly weaker than (18) with $\Lambda:=\Lambda_{1}$. It is a bit trivial, but this allows to expose clearly the main differences between (8) and (18).

Example 3 Assume that $(X,\|\cdot\|, \eta)$ is a real quasi-normed space. Let $Y=X^{2}$ and define $d: Y^{2} \rightarrow \mathbb{R}_{+}$by

$$
d\left(\left(x_{1}, x_{2}\right),\left(y_{1}, y_{2}\right)\right)=\sum_{i=1}^{2}\left\|x_{i}-y_{i}\right\|, \quad\left(x_{1}, x_{2}\right),\left(y_{1}, y_{2}\right) \in Y .
$$

Clearly, $(Y, d, \eta)$ is a b-metric space.

Fix $\tau_{1}, \tau_{2} \in X^{E}$ and define the operator $\mathcal{T}: Y^{E} \rightarrow Y^{E}$ by

$$
(\mathcal{T} \phi)(t)=\left(\tau_{1}(t), \phi_{1}(t)+\tau_{2}(t)\right), \quad t \in E,
$$

for all $\phi=\left(\phi_{1}, \phi_{2}\right) \in Y^{E}$. Then

$$
\begin{aligned}
d((\mathcal{T} \phi)(t),(\mathcal{T} \mu)(x)) & =d\left(\left(\tau_{1}(t), \phi_{1}(t)+\tau_{2}(t)\right),\left(\tau_{1}(t), \mu_{1}(t)+\tau_{2}(t)\right)\right) \\
& \leq d(\phi(t), \mu(t)), \quad t \in E,
\end{aligned}
$$

for every $\phi=\left(\phi_{1}, \phi_{2}\right), \mu=\left(\mu_{1}, \mu_{2}\right) \in Y^{E}$. Therefore, with any $\Lambda_{1}: \mathbb{R}_{+}^{E} \rightarrow \mathbb{R}_{+}^{E}$ such that $\Lambda_{1} \delta \geq \delta$ for $\delta \in \mathbb{R}_{+}{ }^{E}$, we have $\Lambda_{1}$-contractivity of $\mathcal{T}$. Next,

$$
\left(\mathcal{T}^{n} \phi\right)(t)=\left(\tau_{1}(t), \tau_{1}(t)+\tau_{2}(t)\right), \quad t \in E, n \in \mathbb{N}, n \geq 2,
$$

for each $\phi=\left(\phi_{1}, \phi_{2}\right) \in Y^{E}$ and we can take $\Lambda_{n} \delta(t)=0$ for $\delta \in \mathbb{R}_{+}{ }^{E}, t \in E$ and $n \in \mathbb{N}$, $n \geq 2$. This means that the second inequality in (8) is fulfilled for every $\varepsilon \in \mathbb{R}_{+}{ }^{E}$.

Fix $\delta \in \mathbb{R}_{+}{ }^{E}$ and $u \in X$ with $\|u\|=1$. Let functions $\phi, \psi \in Y^{E}$ be defined by:

$$
\phi(t)=(\delta(t) u, 0), \quad \psi(t)=(0,0), \quad t \in E .
$$

Clearly,

$$
\begin{aligned}
(\mathcal{T} \phi)(t) & =\left(\tau_{1}(t), \delta(t) u+\tau_{2}(t)\right), \\
(\mathcal{T} \psi)(t) & =\left(\tau_{1}(t), \tau_{2}(t)\right), \quad t \in E, \\
d(\phi(t), \psi(t)) & =\|\delta(t) u\|=\delta(t), \quad t \in E .
\end{aligned}
$$

Assume now that $\mathcal{T}$ is $\Lambda_{1}$-contractive with some $\Lambda_{1}: \mathbb{R}_{+}{ }^{E} \rightarrow \mathbb{R}_{+}{ }^{E}$. Then

$$
\delta(t)=\|\delta(t) u\|=d((\mathcal{T} \phi)(t),(\mathcal{T} \psi)(t)) \leq \Lambda_{1} \delta(t), \quad t \in E .
$$


Thus we have shown that

$$
\Lambda_{1} \delta \geq \delta, \quad \delta \in \mathbb{R}_{+}{ }^{E} .
$$

Consequently, by an easy induction, for each $n \in \mathbb{N}$, we obtain

$$
\Lambda_{1}{ }^{n} \delta \geq \delta, \quad \delta \in \mathbb{R}_{+}{ }^{E},
$$

whence

$$
\sum_{i=0}^{\infty}\left(\Lambda_{1}^{i} \delta\right)(t)=\infty, \quad t \in E, \delta \in \mathbb{R}_{+}{ }^{E}, \delta(t) \neq 0
$$

\section{Ulam stability}

Now, we present a simple Ulam stability result that can be derived from our considerations in the previous sections. To this end, we need the following hypothesis.

$$
\begin{aligned}
& \text { (H1) } j \in \mathbb{N}, L_{i}: E \rightarrow \mathbb{R}_{+} \text {for } i=1, \ldots, j, \Phi: E \times Y^{j} \rightarrow Y, \text { and } \\
& \begin{aligned}
d\left(\Phi\left(t, w_{1}, \ldots, w_{j}\right), \Phi\left(t, z_{1}, \ldots, z_{j}\right)\right) & \leq \sum_{k=1}^{j} L_{k}(t) d\left(w_{k}, z_{k}\right) \\
& t \in E,\left(w_{1}, \ldots, w_{j}\right),\left(z_{1}, \ldots, z_{j}\right) \in Y^{j} .
\end{aligned}
\end{aligned}
$$

The following corollary can be easily deduced from Corollary 13 .

Corollary 15 Let hypothesis (H1) be valid, $f_{1}, \ldots, f_{j} \in E^{E}, \varphi \in Y^{E}, \varepsilon \in \mathbb{R}_{+}{ }^{E}$ and

$$
\begin{aligned}
& d\left(\Phi\left(t, \varphi\left(f_{1}(t)\right), \ldots, \varphi\left(f_{j}(t)\right)\right), \varphi(t)\right) \leq \varepsilon(t), \quad t \in E, \\
& \varepsilon^{\star}(t):=\sum_{i=0}^{\infty}\left(\left(\Lambda^{i} \varepsilon\right)(t)\right)^{\xi}<\infty, \quad t \in E,
\end{aligned}
$$

where $\Lambda: \mathbb{R}_{+}{ }^{E} \rightarrow \mathbb{R}_{+}{ }^{E}$ is given by

$$
(\Lambda \delta)(t)=\sum_{k=1}^{j} L_{k}(t) \delta\left(f_{k}(t)\right), \quad \delta \in \mathbb{R}_{+}^{E}, t \in E .
$$

Then the limit

$$
\psi(t):=\lim _{n \rightarrow \infty}\left(\mathcal{T}^{n} \varphi\right)(t)
$$

exists for each $t \in E$, with $\mathcal{T}$ given by:

$$
(\mathcal{T} \varphi)(t):=\Phi\left(t, \varphi\left(f_{1}(t)\right), \ldots, \varphi\left(f_{j}(t)\right)\right), \quad \varphi \in Y^{E}, t \in E,
$$

and $\psi \in Y^{E}$, defined by (28), is a solution of the functional equation

$$
\Phi\left(t, \psi\left(f_{1}(t)\right), \ldots, \psi\left(f_{j}(t)\right)\right)=\psi(t), \quad t \in E,
$$

such that

$$
d(\varphi(t), \psi(t)) \leq\left(4 \varepsilon^{\star}(t)\right)^{1 / \xi}=: \bar{\varepsilon}(t), \quad t \in E .
$$

Moreover, if

$$
\liminf _{n \rightarrow \infty}\left(\Lambda^{n} \bar{\varepsilon}\right)(t)=0, \quad t \in E,
$$


then $\psi$ is the unique function in $Y^{E}$ that satisfies (29) and (30).

Proof Clearly, inequalities (26) and (27) imply (18). Next, (25) means that $\mathcal{T}$ is $\Lambda$-contractive. Note yet that $\mathcal{C}_{0}$ holds (see Remark 3 ), whence (21) is valid. Consequently, by Corollary 13 with $\mathcal{C}=Y^{E}$, the function $\psi$ defined by (28) is a fixed point of $\mathcal{T}$ (that is a solution of (29)) satisfying (30) (take $n=0$ in (20)).

Further, (31) implies (22) for every $t \in E$ with $\gamma=\bar{\varepsilon}$. So, by (B), $\psi$ is the unique function in $Y^{E}$ that satisfies (29) and (30).

Stability of functional equations of form (29) (or related to it) has already been studied by several authors and for further information we refer to $[1,5,8]$. A very particular case of (29) is the linear functional equation of the form

$$
\phi(t)=\sum_{i=1}^{j} L_{i}(t) \phi\left(f_{i}(t)\right),
$$

with $j \in \mathbb{N}, f_{1}, \ldots, f_{j} \in E^{E}, L_{1}, \ldots, L_{j}: E \rightarrow \mathbb{R}_{+}$and for functions $\phi \in Y^{E}$ (provided $Y$ is a real linear space); some recent results concerning stability of less general cases of it can be found in $[8,17,18,21]$. A result similar to Corollary 15 can also be obtained for the functional equation

$$
\phi(t)=\max _{i=1, \ldots, j} L_{i}(t) \phi\left(f_{i}(t)\right),
$$

with $Y=E=\mathbb{R}$ and $d(x, y)=|x-y|^{p}$ for $x, y \in \mathbb{R}$, with some fixed $p \in[1, \infty)$ (see Remark 4). Then we take $\mathcal{T}: Y^{E} \rightarrow Y^{E}$ given by

$$
(\mathcal{T} \varphi)(t)=\max _{i=1, \ldots, j} L_{i}(t) \varphi\left(f_{i}(t)\right), \quad \varphi \in Y^{E}, t \in E,
$$

and it is easy to show that such $\mathcal{T}$ is $\Lambda$-contractive with $\Lambda: \mathbb{R}_{+}{ }^{E} \rightarrow \mathbb{R}_{+}{ }^{E}$ defined by the formula:

$$
(\Lambda \xi)(t)=\max _{i=1, \ldots, j}\left|L_{i}(t)\right|^{p} \xi\left(f_{i}(t)\right), \quad \xi \in \mathbb{R}_{+}^{E}, t \in E
$$

To this end it is enough to prove by a simple induction that

$$
\left|\max _{i=1, \ldots, j} x_{i}-\max _{i=1, \ldots, j} y_{i}\right| \leq \max _{i=1, \ldots, j}\left|x_{i}-y_{i}\right|, \quad x_{i}, y_{i} \in \mathbb{R}, i=1, \ldots, j,
$$

using the inequality

$$
\left|\max \left\{x_{1}, x_{2}\right\}-\max \left\{y_{1}, y_{2}\right\}\right| \leq \max \left\{\left|x_{1}-y_{1}\right|,\left|x_{2}-y_{2}\right|\right\}, \quad x_{1}, x_{2}, y_{1}, y_{2} \in \mathbb{R},
$$

which is very easy to check, because only the case

$$
\max \left\{x_{1}, x_{2}\right\}=x_{1}, \quad \max \left\{y_{1}, y_{2}\right\}=y_{2}
$$

must be verified and then we have the following two situations:

$$
0 \leq x_{1}-y_{2} \leq x_{1}-y_{1}, \quad 0 \leq y_{2}-x_{1} \leq y_{2}-x_{2} .
$$




\section{Approximate eigenvalues}

Assume additionally that $(Y,\|\cdot\|, \eta)$ is a real quasi-normed space and $d(x, y)=\|x-y\|$ for $x, y \in Y$. Clearly, then $Y^{E}$ is also a real liner space with the operations defined pointwise in the usual way:

$$
(\xi+\eta)(t):=\xi(t)+\eta(t), \quad(\alpha \nu)(t):=\alpha \nu(t), \quad v, \mu \in Y^{E}, t \in E, \alpha \in \mathbb{R} .
$$

The next corollary is an example of a result (that can be derived from the previous sections) concerning approximate eigenvalues of some linear operators on $Y^{E}$. Actually the assumption of linearity of the operators is not necessary in the proof, but the notion of eigenvalue might be ambiguous without it (see, e.g., [26]) and therefore we confine only to the linear case.

Corollary 16 Let $\gamma \in \mathbb{R} \backslash\{0\}, \Lambda_{0}: \mathbb{R}_{+}{ }^{E} \rightarrow \mathbb{R}_{+}{ }^{E}$, and $T_{0}: Y^{E} \rightarrow Y^{E}$ be $\Lambda_{0}$-contractive and linear. Suppose that there are $h \in Y^{E}$ and $\varepsilon \in \mathbb{R}_{+}{ }^{E}$ such that

$$
\begin{aligned}
& \left\|\left(T_{0} h\right)(t)-\gamma h(t)\right\| \leq \varepsilon(t), \quad t \in E, \\
& \varepsilon^{\star}(t):=\sum_{i=0}^{\infty}\left(\left(\Lambda^{i} \varepsilon\right)(t)\right)^{\xi}<\infty, \quad t \in E,
\end{aligned}
$$

and (21) is valid, where $\Lambda \delta:=|\gamma|^{-1}\left(\Lambda_{0} \delta\right)$ for $\delta \in \mathbb{R}_{+}{ }^{E}$. Then $\gamma$ is an eigenvalue of $T_{0}$, the limit

$$
\psi(t):=\lim _{n \rightarrow \infty}\left(T_{0}^{n}\left(\gamma^{-n+1} h\right)\right)(t)
$$

exists for every $t \in E$, and the function $\psi_{0} \in Y^{E}$, given by

$$
\psi_{0}(t):=\gamma^{-1} \psi(x), \quad t \in E,
$$

is an eigenvector of $T_{0}$, with the eigenvalue $\gamma$, and

$$
\left\|\psi_{0}(t)-h(t)\right\| \leq|\gamma|^{-1} \varepsilon^{*}(t), \quad t \in E .
$$

Proof Let $\varphi:=\gamma h$ and $\mathcal{T}: Y^{X} \rightarrow Y^{X}$ be given by:

$$
(\mathcal{T} v)(t)=\left(T_{0}\left(\gamma^{-1} v\right)\right)(t), \quad v \in Y^{E}, t \in E .
$$

Then, in view of the $\Lambda_{0}$-contractivity and linearity of $T_{0}$, for every $\mu, v \in Y^{E}$ and $\delta \in \mathbb{R}_{+}{ }^{E}$ with $d(\mu, v) \leq \delta$ we have

$$
\begin{aligned}
d(\mathcal{T} \mu, \mathcal{T} v) & =d\left(T_{0}\left(\gamma^{-1} \mu\right), T_{0}\left(\gamma^{-1} \nu\right)\right) \\
& \leq\left|\gamma^{-1}\right| d\left(T_{0} \mu, T_{0} v\right) \leq\left|\gamma^{-1}\right|\left(\Lambda_{0} \delta\right),
\end{aligned}
$$

which means that $\mathcal{T}$ is $\Lambda$-contractive. Next, we can write (32) in the form

$$
d((\mathcal{T} \varphi)(t), \varphi(t)) \leq \varepsilon(t), \quad t \in E .
$$

Hence, by Corollary 13 (with $\mathcal{C}=Y^{E}$ ), the limit (19) exists for each $t \in E$ and the function $\psi: E \rightarrow Y$, so defined, is a fixed point of $\mathcal{T}$ with

$$
d(\psi(t), \varphi(t)) \leq \varepsilon^{*}(t), \quad t \in E .
$$

Write $\psi_{0}:=\gamma^{-1} \psi$. Now, it is easily seen that $T_{0} \psi_{0}=\mathcal{T} \psi=\psi=\gamma \psi_{0}$, (34) is equivalent to (36), and (19) yields (33). 
Clearly, under suitable additional assumptions in Corollary 16, we can deduce from Corollary 13 some statements on the uniqueness of $\psi$, and consequently of the uniqueness of $\psi_{0}$.

Given $\varepsilon \in \mathbb{R}_{+}{ }^{E}$, let us introduce the following definition: $\gamma \in \mathbb{R} \backslash\{0\}$ is an $\varepsilon$-eigenvalue of a linear operator $T_{0}: Y^{E} \rightarrow Y^{E}$ provided there exists $h \in Y^{E}$ such that $\left\|T_{0} h-\gamma h\right\| \leq \varepsilon$.

Corollary 16 yields the following simple result.

Corollary 17 Let $\Lambda_{0}: \mathbb{R}_{+}{ }^{E} \rightarrow \mathbb{R}_{+}{ }^{E}, T_{0}: Y^{E} \rightarrow Y^{E}$ be $\Lambda_{0}$-contractive and linear, and $\varepsilon \in \mathbb{R}_{+}{ }^{E}$. Then every $\varepsilon$-eigenvalue $\gamma \in \mathbb{R} \backslash\{0\}$ of $T_{0}$ such that

$$
\varepsilon^{\star}(t):=\sum_{i=0}^{\infty}\left(\left(\Lambda^{i} \varepsilon\right)(t)\right)^{\xi}<\infty, \quad t \in E,
$$

$\left(\right.$ with $\Lambda \delta:=|\gamma|^{-1}\left(\Lambda_{0} \delta\right)$ for $\left.\delta \in \mathbb{R}_{+}{ }^{E}\right)$ and (21) holds, is an eigenvalue of $T_{0}$.

Open Access This article is licensed under a Creative Commons Attribution 4.0 International License, which permits use, sharing, adaptation, distribution and reproduction in any medium or format, as long as you give appropriate credit to the original author(s) and the source, provide a link to the Creative Commons licence, and indicate if changes were made. The images or other third party material in this article are included in the article's Creative Commons licence, unless indicated otherwise in a credit line to the material. If material is not included in the article's Creative Commons licence and your intended use is not permitted by statutory regulation or exceeds the permitted use, you will need to obtain permission directly from the copyright holder. To view a copy of this licence, visit http://creativecommons.org/licenses/by/4.0/.

\section{References}

1. Agarwal, R.P., Xu, B., Zhang, W.: Stability of functional equations in single variable. J. Math. Anal. Appl. 288, 852-869 (2003)

2. Boriceanu, M., Bota, M., Petruşel, A.: Multivalued fractals in $b$-metric spaces. Cent. Eur. J. Math. 8(2), 367-377 (2010)

3. Brzdęk, J., Cădariu, L., Ciepliński, K.: Fixed point theory and the Ulam stability. J. Funct. Spaces Art. ID 829419, 16 pp (2014)

4. Brzdęk, J., Ciepliński, K.: A fixed point approach to the stability of functional equations in nonArchimedean metric spaces. Nonlinear Anal. 74(18), 6861-6867 (2011)

5. Brzdęk, J., Ciepliński, K., Leśniak, Z.: On Ulam's type stability of the linear equation and related issues. Discrete Dyn. Nat. Soc. 536791, 14 (2014)

6. Brzdęk, J., Chudziak, J., Páles, Z.: A fixed point approach to stability of functional equations. Nonlinear Anal. 74, 6728-6732 (2011)

7. Brzdęk, J., Karapınar, E., Petruşel, A.: A fixed point theorem and the Ulam stability in generalized dq-metric spaces. J. Math. Anal. Appl. 467, 501-520 (2018)

8. Brzdęk, J., Popa, D., Raşa, I., Xu, B.: Ulam Stability of Operators, Mathematical Analysis and its Applications, vol. 1. Academic Press, Elsevier (2018)

9. Cădariu, L., Găvruţa, L., Găvruţa, P.: Fixed points and generalized Hyers-Ulam stability. Abstr. Appl. Anal. Art. ID 712743, 10 pp (2012)

10. Czerwik, S.: Contraction mappings in $b$-metric spaces. Acta Math. Univ. Ostrav. 1(1), 5-11 (1993)

11. Czerwik, S.: Nonlinear set-valued contraction mappings in $b$-metric spaces. Atti Sem. Math. Fis. Univ. Modena 46, 263-276 (1998)

12. Deza, M.M., Deza, E.: Encyclopedia of Distances. Springer, Berlin (2009)

13. Dung, N.V., Hang, V.T.L.: The generalized hyperstability of general linear equations in quasi-Banach spaces. J. Math. Anal. Appl. 462, 131-147 (2018)

14. Feng, Y., Liu, S.: Fixed point theorems for multi-valued contractive mappings and multi-valued Caristi type mappings. J. Math. Anal. Appl. 317, 103-112 (2006)

15. Heinonen, J.: Lectures on Analysis on Metric Spaces. Universitext, Springer (2001)

16. Hyers, D.H., Isac, G., Rassias, Th.M.: Stability of Functional Equations in Several Variables. Birkhäuser, Boston (1998) 
17. Jung, S.-M., Rassias, M.Th.: A linear functional equation of third order associated to the Fibonacci numbers. Abstr. Appl. Anal. 2014, 137468 (2014)

18. Jung, S.-M., Popa, D., Rassias, M.Th.: On the stability of the linear functional equation in a single variable on complete metric groups. J. Glob. Optim. 59, 165-171 (2014)

19. Kadelburg, Z., Radenović, S.: On generalized metric spaces: a survey. TWMS J. Pure Appl. Math. 5(1), 3-13 (2014)

20. Maligranda, L., Tosio Aoki (1910-1989), in: Proceedings of the International Symposium on Banach and Function Spaces II, Kitakyushu, Japan, 2006, Yokohama Publishers. pp. 1-23 (2008)

21. Mortici, C., Rassias, M.Th., Jung, S.-M.: On the stability of a functional equation associated with the Fibonacci numbers. Abstr. Appl. Anal. 2014, 6 (2014)

22. Moszner, Z.: Stability has many names. Aequationes Math. 90, 983-999 (2016)

23. Nashine, H.K., Ibrahim, R.W., Rhoades, B.E., Pant, R.: Unified Feng-Liu type fixed point theorems solving control problems. Rev. R. Acad. Cienc. Exactas Fís. Nat. Ser. A Mat. RACSAM 115(1), 17 (2021)

24. Paluszyński, M., Stempak, K.: On quasi-metric and metric spaces. Proc. Am. Math. Soc. 137(12), 43074312 (2009)

25. Parvaneh, V., Hussain, N., Kadelburg, Z.: Generalized Wardowski type fixed point theorems via $\alpha$ admissible FG-contractions in b-metric spaces. Acta Math. Sci. 36B(5), 1445-1456 (2016)

26. Santucci, P., Väth, M.: On the definition of eigenvalues for nonlinear operators. Nonlinear Anal. 40, 565-576 (2000)

27. Schroeder, V.: Quasi-metric and metric spaces. Conform. Geom. Dyn. 10, 355-360 (2006)

28. Van Dung, N.: A new approach to fixed point theorems in compact 2-generalized metric spaces. Rev. R. Acad. Cienc. Exactas Fís. Nat. Ser. A Mat. RACSAM 115(1), 8 (2021)

29. Wardowski, D.: Fixed point theory of a new type of contractive mappings in complete metric spaces. Fixed Point Theory Appl. 2012, 94 (2012)

Publisher's Note Springer Nature remains neutral with regard to jurisdictional claims in published maps and institutional affiliations. 